\title{
PARTICIPAÇÃO DA FAMÍLIA NO TRABALHO FISIOTERAPÊUTICO EM CRIANÇAS COM PARALISIA CEREBRAL
}

\section{PARTICIPATION OF THE FAMILY IN PHYSICAL THERAPY TREATMENT OF CHILDREN WITH CEREBRAL PALSY}

Franciele Leiliane Sari *

Sonia Silva Marcon **

Sari FL, Marcon SS. Participação da Família no Trabalho Fisioterapêutico em Crianças com Paralisia Cerebral. Rev Bras Crescimento Desenvolvimento Hum. 2008; 18(3): 229-239

\section{Resumo:}

Estudo descritivo exploratório realizado com mães de crianças menores de cinco anos, com diagnóstico de Paralisia Cerebral, com o objetivo de verificar a participação dos pais no processo de reabilitação das crianças e suas expectativas em relação a este tratamento. Os dados foram coletados no mês de outubro de 2006 por meio de entrevistas semiestruturadas junto a 28 mães de crianças matriculadas em uma escola de reabilitação. As mães individualmente (39\%) ou em conjunto com os pais (32\%) ou outros familiares são as principais responsáveis pelo desenvolvimento de atividades fisioterapêuticas no domicílio. Estas são realizadas diariamente (36\%) ou duas a três vezes por semana (39\%), normalmente à noite e utilizando estímulos diferenciados (96\%). A maioria (79\%) refere conseguir realizar todas as atividades propostas, sendo as principais dificuldades relacionadas à não aceitação por parte da criança (54\%) e à falta de tempo (21\%). As expectativas são que a criança consiga andar sem apoio (46\%) e que tenha independência motora (25\%). Embora em graus variados, na maioria das vezes, os profissionais podem contar com a ajuda da família na oferta de estímulos às crianças. As mães que já passaram por várias sessões de orientação, se mostram mais seguras para a realização das atividades fisioterapêuticas no domicílio, revelando a importância da interação com a família. Ao profissional cabe esclarecer a família sobre a importância dos exercícios mesmo quando o prognóstico é limitado, pois a evolução da criança pode surpreender e, além disso, esta é uma oportunidade para desenvolver/fortalecer o vínculo entre a família e a criança.

Palavras-chave: Fisioterapia; paralisia cerebral; relações profissional-família.

\footnotetext{
* Fisioterapeuta da Associação Norte Paranaense de Reabilitação - ANPR, Escola de Educação Especial “Albert-Sabin”.

** Enfermeira. Doutora em Filosofia da Enfermagem. Coordenadora do Mestrado em Enfermagem da UEM. Coordenadora do NEPAAF.

Correspondência para: Sonia Silva Marcon. Rua Jailton Saraiva, 526. Jardim América. Maringá - PR. CEP: 87045-300. E-mail: soniasilva.marcon@gmail.com
} 


\begin{abstract}
:
This is a descriptive and exploratory study carried out with mothers of children aged five years or less, with diagnosis of Cerebral Palsy. It aims to investigate parents' participation in the process of children's rehabilitation and their expectation regarding the treatment. Data were collected in the month of October 2006 through semi-structured interviews conducted with 28 mothers of children enrolled in a rehabilitation school. The mothers, individually (39\%) or together with the fathers (32\%) or other family members, are responsible for the development of physiotherapy activities at home. These are performed on a daily basis (36\%) or two to three times a week (39\%), usually at night and using differentiated stimuli (96\%). Most mothers (79\%) stated they are able to conduct all the proposed activities, and the main difficulties are related to non-acceptance on the part of the child (54\%) and lack of time (21\%). The expectation is that the child becomes able to walk without support (46\%) and that he/she has motor independence (25\%). Although in varied degrees, most of the time the professionals can count on the family's help concerning the offer of stimuli to the children. The mothers who already had several orientation sessions feel more prepared to conduct the physiotherapy activities at home. This reveals the importance of the interaction with the family. The professional needs to explain to the family the importance of the exercises even when the prognostic is limited, because the child's evolution can be surprising and, besides, this is an opportunity to develop/strengthen the bond between the family and child.
\end{abstract}

Key words: Physiotherapy; cerebral palsy; professional-family relationship.

\section{INTRODUÇÃO}

A atuação e colaboração familiar é extremamente importante para qualquer profissional que trabalhe com crianças que apresentam algum tipo de alteração no desenvolvimento motor e, em especial, quando se trata de crianças com Paralisia Cerebral. Nesse sentido, tem-se observado respostas positivas quando os familiares atuam de forma a promover uma melhor evolução da criança.

A expressão Paralisia Cerebral (doravante PC) é definida como "seqüela de uma agressão encefálica, que se caracteriza, primordialmente, por um transtorno persistente, mas não invariável, do tono, da postura e do movimento, que aparece na primeira infância e que não só é diretamente secundário a esta lesão não evolutiva do encéfalo, senão devido, também, à influência que tal lesão exerce na maturação neurológica”. ${ }^{1}$

Em países em desenvolvimento, a incidência desta doença é maior do que nos países desenvolvidos, observando-se índices de 7:1000. No Brasil, estima-se uma prevalência de 30000 a 40000 casos novos por ano. ${ }^{2}$

Com base no modelo de classificação proposto pela Organização Mundial de Saúde, esta enfermidade pode apresentar consequências variadas. No que se refere à função de órgãos e sistemas, a paralisia cerebral geralmente interfere no funcionamento do sistema músculo-esquelético. Neste nível, as características associadas a esta patologia incluem distúrbios de tônus muscular, postura e movimentação voluntária. ${ }^{2}$

O comprometimento neuromotor desta doença pode envolver partes distintas do cor- 
po, resultando em classificações topográficas específicas (quadriplegia, hemiplegia e diplegia). Também pode ser classificada com base nas alterações clínicas do tono muscular e no tipo de desordem do movimento podendo produzir o tipo espástico, discinético ou atetóide, atáxico, hipotônico e misto. A gravidade do comprometimento neuromotor de uma criança com paralisia cerebral pode ser caracterizada como leve, moderada ou severa, baseada no meio de locomoção da criança. ${ }^{3}$

Estas classificações servem a um propósito de descrição e caracterização da lesão, não fornecendo informação sobre as consequências desta enfermidade na rotina diária da criança. ${ }^{2,3}$ Esta doença ocorre no período em que a criança apresenta ritmo acelerado de desenvolvimento, podendo comprometer o processo de aquisição de habilidades. Tal comprometimento pode interferir na função, dificultando o desempenho de atividades frequentemente realizadas por crianças com desenvolvimento normal. ${ }^{2}$

Os pacientes com PC devem ser tratados por uma equipe multidisciplinar, na qual o principal enfoque terapêutico é, sem dúvida, o fisioterápico e, nestes casos, diferentes métodos utilizados em fisioterapia são empregados de acordo com o quadro clínico. Dentre eles, utilizam-se, principalmente, três métodos: o de Bobath, que se baseia na inibição dos reflexos primitivos e dos padrões patológicos de movimentos; o de Phelps, que se baseia na habilitação por etapas, dos grupos musculares, até chegar à independência motora e a praxias complexas e o de Kabat, que se baseia na utilização de estímulos proprioceptivos facilitadores das respostas motoras, partindo de respostas reflexas, e chegando à motricidade voluntária. ${ }^{1}$
A intervenção fisioterapêutica em neurologia infantil é frequentemente conduzida a partir da relação entre a postura e o movimento da criança. O controle postural é responsável pela resistência à ação da gravidade e pela manutenção do equilíbrio do corpo durante os movimentos voluntários que exigem estabilidade de membros, tronco e corpo. ${ }^{4}$

$\mathrm{O}$ atendimento fisioterapêutico sempre deve levar em consideração as etapas do desenvolvimento motor normal, utilizando vários tipos de estimulação sensitiva e sensorial, haja vista que este tipo de desenvolvimento depende da tarefa e das exigências do ambiente, havendo, portanto, a necessidade de manter estreitas relações com o desenvolvimento visual, cognitivo e da fala. Dessa forma, o comportamento da criança se modifica, tornandose mais intencional à medida que se processa a maturação do seu sistema nervoso. ${ }^{5}$

Como já foi ressaltado anteriormente, a paralisia cerebral é uma patologia que implica em limitações motoras graves para a criança, tornando-a muito dependente. Sabe-se que apenas as sessões de fisioterapia realizadas em ambulatório são insuficientes para os estímulos de desenvolvimento necessários a essa criança. Tal fato torna fundamental o papel da família no processo de reabilitação.

Mediante a rotina dos atendimentos fisioterapêuticos a crianças com paralisia cerebral, foi possível observar que os pais têm dificuldades para realizar as orientações propostas em seu domicílio, não interagindo de maneira completa com o tratamento do seu filho. Questiona-se, assim, se tal comportamento está relacionado com a aceitação da patologia da criança, com a interação em relação ao trabalho do profissional, ou simplesmente pela 
falta de esclarecimento em relação à importância do seu papel para a reabilitação da criança.

De fato, essas crianças necessitam de um cuidado especial devido a todos os comprometimentos, mas a família também necessita de atenção e deve ser incluída no plano terapêutico, uma vez que, em muitos casos, ela demonstra significativa falta de informação relacionada com a condição da criança e desorientação sobre a forma como pode e deve agir para melhor ajudar o filho.

Esta condição é particularmente acentuada nos primeiros tempos após o diagnóstico, ocasião em que se torna imprescindível a intervenção do profissional, a fim de oferecer suporte e permitir que a família participe ativamente das decisões relacionadas com o tratamento de seu filho. ${ }^{6}$

Este, por sua vez, deve ser iniciado o mais precocemente possível - devido à maior plasticidade neuromotora, já que o propósito é contribuir positivamente para o melhor desenvolvimento motor da criança. ${ }^{6}$

O profissional deve sempre fundamentar o seu papel, qual seja, oferecer suporte e orientação para os pais, de forma que estes possam ajudar no tratamento e não se utilizar das orientações com o intuito de torná-los terapeutas experientes ou substitutos deste profissional. ${ }^{6}$

Assim, o objetivo do estudo é verificar a participação dos pais no processo de reabilitação de crianças com paralisia cerebral e suas expectativas em relação a este tratamento.

\section{MÉTODO}

Estudo descritivo exploratório realizado junto a famílias de crianças com paralisia cerebral, matriculadas na Escola de Educação Especial Albert Sabin, vinculada à Associação Norte Paranaense de Reabilitação (ANPR).

O estudo foi realizado junto ao serviço de fisioterapia da entidade, que presta atendimento a 220 crianças e jovens na faixa etária de 0 a 29 anos, que possuem as mais variadas patologias. Dentre essas patologias, a paralisia cerebral é a mais frequente.

Os dados foram coletados durante o mês de outubro e novembro de 2006 por meio de entrevistas semi-estruturadas com os familiares. O instrumento utilizado para a coleta de dados foi um questionário semi-estruturado, elaborado pela própria pesquisadora a partir dos objetivos do estudo. Este instrumento foi submetido à avaliação aparente e de conteúdo por dois profissionais, um da área de fisioterapia e outro que trabalha com famílias.

Os informantes foram os pais e/ou responsáveis pelas crianças selecionadas para o estudo. Os critérios de inclusão adotados na seleção das crianças que fizeram parte do estudo foram: a criança ter idade igual ou menor que cinco anos, ter sido diagnosticada com o quadro clínico de paralisia cerebral em fase de desenvolvimento das habilidades motoras, estar sendo atendida pelo serviço de fisioterapia da instituição e cujos pais ou responsáveis tivessem sido orientados pelo profissional fisioterapeuta no primeiro semestre de 2006. A localização das crianças que atendiam a estes critérios foi realizada através de consulta ao arquivo do setor de fisioterapia da instituição.

No total foram identificadas 31 crianças que atendiam aos critérios estabelecidos, mas três mães não aceitaram participar do estudo, de forma que a população efetivamente estudada está constituída 28 familiares de 
crianiniciada após a avaliação e a aprovação do projeto pelo comitê de ética da instituição signatária.

\section{RESULTADOS}

\section{Características das crianças}

Das 28 crianças em estudo, a maioria (61\%) encontra-se na faixa etária de um a três anos e as demais, tem entre três a cinco anos; mais da metade é do sexo masculino (54\%) e procedentes do próprio município (54\%). As demais crianças são procedentes de cidades vizinhas e que fazem parte da região metropolitana de Maringá, sendo seis crianças de Paiçandu (21\%), três de Sarandi (11\%) e uma das cidades de Ourizona, Marialva, Mandaguaçu e Iguatemi. A maioria (68\%) nasceu de parto cesárea.

A maioria das crianças (68\%) apresenta algum tipo de problema associado à patologia de base. Observou-se que o comprometimento mais freqüente é a epilepsia, com 12 casos (43\%), sendo que desses apenas dois não tem associação com outros acometimentos, tais como: déficit visual, hidrocefalia, microcefalia, estrabismo e déficit auditivo.

O estrabismo aparece em quatro casos (14\%) e, entre estes casos, dois também estão associados a outros acometimentos como hidrocefalia. Os déficits visuais comprometem oito crianças (29\%) e estão associados à epilepsia, microcefalia e hidrocefalia. Duas crianças apresentam comprometimento auditivo, sendo que uma delas não tem outros problemas associados. A hidrocefalia está presente em cinco crianças (18\%) e apenas uma não apresenta outros comprometimentos. Já a microcefalia está presente em três crianças (11\%). Uma criança apresenta apnéia e outra catarata, ambos os casos associados a outros comprometimentos já citados anteriormente.

Ao se investigar as concepções das mães sobre a etiologia da paralisia cerebral, observou-se que a maioria delas (68\%) acredita que o problema é decorrente de intercorrências durante o parto ou no período peri-natal, sendo que todas referiram a presença de anóxia neonatal. É interessante observar que dez mães (36\%) acreditam que a PC tem relação com problemas vivenciados ainda na gestação, tais como prematuridade (quatro casos), infecções e pré-eclampsia (dois casos cada) e diabetes melitus e sangramento (um caso cada).

Além disso, nove crianças (32\%) apresentaram problemas após o nascimento, sendo que seis tiveram infecção e outras três, intervenções cirúrgicas com complicações.

\section{O trabalho fisioterapêutico}

Em relação ao tempo que realiza fisioterapia, constatou-se que três crianças (11\%) realizam fisioterapia há menos de um ano e correspondem àquelas com idade inferior a um ano e seis meses. Onze crianças (39\%) realizam fisioterapia entre um e três anos e 14 crianças (50\%) de três a cinco anos. Portanto, considerando a idade das crianças em estudo, observa-se que a maioria delas iniciou o tratamento fisioterapêutico precocemente.

Ao se investigar quem na família recebeu orientações fisioterapêuticas, observou-se que em 96\% dos casos foram as mães. Em quatro casos outros membros também receberam. Apenas em um caso o pai foi o único a receber orientações. 
A realização de atividades fisioterapêuticas em casa normalmente fica a cargo das mães, sendo que em 11 casos (39\%) elas são as únicas responsáveis por esta atividade, em nove (32\%) este encargo é dividido com o pai e em seis (21\%) com outro familiar. Em dois casos o pai é o único responsável pelo desenvolvimento desta atividade no domicílio.

Na concepção da maioria das mães entrevistadas (92\%) são elas que estão melhor preparadas para a realização das atividades fisioterapêuticas no domicílio em, um caso refere ser a avó e em outro a tia. É relevante observar que duas mães entrevistadas relataram que o pai está melhor preparado, sendo ele o responsável por tal função.

Quando questionado com quem a criança mais gosta de fazer fisioterapia, obteve-se maior freqüência de respostas indicando as mães (61\%), seguido por mãe e/ou pai, (quatro casos), apenas com o pai (um caso), outro familiar no caso avó e tia, (dois casos), e uma mãe referiu que a criança não se manifesta.

Todas as entrevistadas já haviam sido orientadas pela fisioterapeuta, sendo que 11 (39\%) foram orientadas uma ou duas vezes, seis foram orientadas três ou quatro vezes, oito foram orientadas cinco a sete vezes, e três foram orientadas oito ou mais vezes.

A maioria das entrevistadas (79\%) afirmou que realizam todas as atividades propostas nas orientações fisioterapêuticas, cinco destacaram que conseguem executar algumas orientações e outras não e uma entrevistada relatou não fazer as atividades propostas em casa, pois "não consigo ver resultados com meu filho” (Paciente 27).

Os motivos apontados para não conseguir realizar algumas das atividades propostas foram: “a criança não deixa” (três casos) e "a mãe não consegue” (dois casos).

As dificuldades que os familiares ou responsáveis sentem em geral para realizar as atividades com as crianças são: a criança não aceita as atividades (54\%); falta de tempo dos pais (21\%); necessidade de maior preparo (três casos); não apresentam dificuldades (três casos) e falta de paciência por não ver resultados (um caso).

Referindo-se ao local da casa onde as atividades fisioterapêuticas são realizadas, constatou-se que com maior freqüência isto ocorre na sala (46\%), seguida de quarto (32\%), quintal ou varanda (14\%) e cozinha (7\%). Quanto ao período do dia em que as atividades são realizadas, observou-se que ele está relacionado com a disponibilidade da mãe e o horário em que a criança frequenta a escola, de forma que a maioria das mães fez referência ao período noturno (36\%), seguido da manhã (25\%) e tarde (21\%). Cinco mães (18\%), por sua vez, mencionaram que realizam as atividades em qualquer período.

Em relação à frequência com que realizam as atividades, uma parcela considerável de mães (36\%) afirmou que elas são realizadas diariamente ou duas a três vezes na semana (39\%); algumas mães, no entanto (14\%) reconheceram que elas são realizadas só uma vez na semana e outras três mães fizeram referência aos finais de semana.

Durante as atividades familiares, também se observou que são aplicados estímulos visuais, cognitivos, sonoros e motores. Assim, 96\% dos entrevistados relataram estimular as crianças com conversas, músicas, DVD, TV, brincadeiras e brinquedos sonoros e pedagógicos. 
Quando os entrevistados foram questionados acerca dos motivos que os levariam a não realizar as atividades, foram destacadas a falta de tempo (79\%) e o dó da criança (14\%). Uma mãe revelou que não realiza tais procedimentos por "não acreditar que dê resultado e por sentir-se despreparada para esta função” (Paciente 27); e outra asseverou que não deixaria de fazer por nenhum motivo (Paciente 11).

\section{Expectativas dos familiares e/ou responsáveis}

A maioria das entrevistadas (96\%) afirmou perceber resultados positivos com o tratamento de fisioterapia. As manifestações sobre estes resultados revelaram que, na percepção das mães, a partir do início das atividades fisioterapêuticas, os filhos apresentaram melhoras representadas pela manifestação de alguns comportamentos não existentes anteriormente, como: sentar (nove), rolar (três), ficar em pé (cinco), engatinhar (quatro), andar (seis), andar com apoio (dois) e pegar objetos (três). É interessante observar que seis mães referiram que com o tratamento fisioterapêutico, "tudo mudou”. Além disso, também foram referidos outros desempenhos, tais com: arrastar (três), controle cervical (nove), atenção e interação (quatro), maior movimentação dos membros (três), troca de passos (três), tônus (dois), equilíbrio (dois), e apoio dos braços em postura de prono (um).

Ao se manifestarem a este respeito, as mães apresentaram depoimentos como os expostos abaixo:

"Melhorou bastante, era bem molinho, não sentava e hoje anda” (Paciente 26).
“Em tudo, não segurava alimento para comer, hoje anda de motoca arrasta e fica sentado" (Paciente 25).

"Era molinho, hoje firma o pescoço, senta e engatinha” (Paciente 16).

Apenas uma das entrevistadas não se mostrou muito otimista com os resultados:

"O resultado foi pouco, mas percebi alguma mudança” (Paciente 27).

As expectativas dos entrevistados em relação ao resultado da fisioterapia para a sua criança são variadas. Uma mãe espera que a criança consiga sentar, duas que a criança consiga se manter sentada, seis que a criança consiga andar com apoio, sete que ela tenha independência e treze que ela consiga andar sem apoio.

\section{DISCUSSÃO}

Os resultados apontam que a realização das atividades fisioterapêuticas, com maior ou menor freqüência, ocorre no domicílio da maioria das crianças em estudo, revelando, assim, que os familiares auxiliam na realização das atividades e na recuperação da criança com paralisia cerebral. Tais constatações ainda evidenciam o reconhecimento da importância da participação efetiva da família a fim de que a criança seja submetida a uma carga maior de estímulos por meio da realização de atividades fisioterápicas também no domicílio.

A interação fisioterapeuta/família deve estar bem estabelecida para que ocorra a troca de informações pertinentes para a definição dos objetivos funcionais possíveis na realidade motora cognitiva e social em que a criança se encontra, contribuindo, assim, para a supera- 
ção de dificuldades vivenciadas diariamente no relacionamento dessa família com a criança. Contudo, esta interação só se torna possível mediante a existência de confiança entre os envolvidos nesse processo. Por esse motivo é importante que desde o início do trabalho com a criança, o terapeuta converse com a família para reconhecer suas dificuldades e seus anseios. ${ }^{8}$

A atuação conjunta profissional e família, resulta em benefícios fundamentais para o desenvolvimento da criança no que concerne ao seu potencial de crescimento e desenvolvimento global. Neste âmbito, é relevante ressaltar que os fatores psicoafetivos e um ambiente familiar estimulante dão suporte para todo o processo de reabilitação, pois é inegável o fato de que a quantidade e qualidade de estímulos recebidos pela criança em casa são insubstituíveis. ${ }^{9}$

As crianças com necessidades especiais carecem da atuação de uma equipe de profissionais, para otimizar o processo de recuperação e estimulação. No entanto, com freqüência, a queixa principal dos familiares das crianças com paralisia cerebral relaciona-se as questões de incapacidades e limitações funcionais, além das desvantagens em relação aos ganhos do desenvolvimento motor, trazendo conseqüências nos diferentes contextos como família, escola e sociedade. ${ }^{10}$

Somente quando o atendimento é focado para a relação mãe-filho, pai-mãe-filho, familiares, escola e comunidade, é que se pode atuar de maneira efetiva. Pois a criança especial exige da família profunda modificação em seus hábitos. ${ }^{9}$

A fisioterapia tem fundamental importância no desenvolvimento motor da criança especial. Observando a faixa etária estabelecida para este estudo (zero a cinco anos), pode-se perceber que $61 \%$ das crianças já realizam fisioterapia há mais de um ano. Neste sentido, os relatos apontaram que, possivelmente, desde o nascimento estas crianças tenham recebido algum tipo de intervenção fisioterapêutica.

Isto é muito importante, pois o primeiro ano de vida da criança é caracterizado por grandes mudanças, o termo desenvolvimento, quando aplicado à evolução da criança significa constante observação no crescimento das estruturas somáticas e aumento das possibilidades de cada um em agir sobre o ambiente. ${ }^{11}$

No caso da lesão cerebral que incide durante a vida intra-uterina, os circuitos corticais e sub-corticais do cérebro são capazes de se reorganizar em sua grande maioria, ao passo que, na criança de idade pré-escolar, os hemisférios cerebrais já assumiram sua função definitiva. ${ }^{5}$

A velocidade do desenvolvimento motor é influenciada, principalmente durante os primeiros 12 e 18 meses de vida, pela maneira com que os pais manuseiam o lactente. Além disso, as condições socioeconômicas da família e a interação entre mãe e lactente são fatores que podem influenciar o desenvolvimento motor. ${ }^{5,12,13}$

Algumas considerações importantes relacionadas ao papel do profissional junto às famílias devem ser destacadas neste aprendizado cooperativo, tais como: estimular e reforçar o vínculo pai-mãe e filho; desenvolver a compreensão e a aceitação da patologia; esclarecer os objetivos do tratamento fisioterapêutico; incentivar a participação na escolha e utilização dos métodos de tratamento; conscientizar os pais da importância de sua partici- 
pação no tratamento e avaliação da evolução; e proporcionar a extensão do tratamento fisioterapêutico em casa, ressaltando os bene-

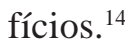

A intervenção fisioterapêutica e o manuseio da criança em casa influenciam diretamente os processos de maturação e recuperação do sistema nervoso, pois tudo o que a criança experimenta e treina provavelmente causa modificações no seu sistema neuromuscular, visto que ambos têm estreita relação. O processo de recuperação deve ser precoce devido a maior plasticidade neuromotora, objetivando melhorar as capacidades funcionais que visam estruturar o ambiente no sentido de favorecer a exploração ativa, e estimular atos tais como controlar a cabeça, rolar, passar para sentado, engatinhar, ficar sobre os joelhos, colocar-se em pé, andar, e apreender e manusear diferentes objetos. Dessa forma, a ênfase recai sobre a criação de um ambiente favorável para a reabilitação, que permita maior interação ativa e voluntária entre a criança, terapeuta/pais e o ambiente. $^{5}$

Considera-se que a idade é um fator importante quando se trata de assumir uma função anteriormente exercida por áreas cerebrais que sofreram lesão. Um ponto de vista largamente aceito, admitia que o cérebro jovem se recuperasse mais facilmente após lesão do que o cérebro adulto, graças à maior plasticidade. ${ }^{5}$

$\mathrm{O}$ fato de que, na maioria dos casos, foram as mães que receberam orientações e que realizam as atividades fisioterapêuticas no domicílio, revela por um lado que a mulher tem uma participação mais efetiva na interação profissional/família durante o processo de reabilitação da criança. E por outro confirma o papel cuidador da mulher na família.
Ademais, é comum que na maioria dos casos, os pais destas crianças se omitam da interação e dos cuidados, deixando a mãe encarregada pela criança. Isto gera sobrecarga, e os pais dessas crianças também tendem ter uma auto-estima baixa e menos sentimentos de competência pessoal. ${ }^{15}$

Contudo, identificamos que a atitude protetora dos familiares, principalmente das mães e a sobrecarga de cuidados direcionados à criança dificultam o processo de intervenção familiar junto à reabilitação. Tal hipótese é confirmada pelo elevado percentual de crianças que não aceitam que o familiar realize as atividades em casa (54\%), e a falta de tempo dos pais (21\%).

Observa-se ainda que as famílias fazem uso de estímulos variados durante as atividades, demonstrando a dedicação com a criança e o compromisso com o processo de reabilitação. Isto é fundamental, pois o ambiente ideal para o bom desenvolvimento infantil é aquele que inclui objetos variados para o bebê explorar, e adultos amorosos, responsivos e sensíveis que conversam frequentemente com a criança durante a realização das atividades. Entre outras contribuições, o ambiente enriquecido pode beneficiar o desenvolvimento e a retenção de uma rede mais elaborada e complexa de conexões neurais. ${ }^{15}$

As manifestações de expectativas da família em relação ao tratamento fisioterapêutico devem ser consideradas à luz do prognóstico da criança que, muitas vezes, não é aceito pela família devido à esperança e à fantasia do "filho sem deficiência”, da confiabilidade a todos os tratamentos indicados. O fato de a maioria das expectativas serem direcionadas à independência da criança pode estar relacio- 
nado com a condição de os familiares não terem sido esclarecidos suficientemente pela equipe de saúde quanto às limitações dessas crianças, ou ainda ao fato de estarem emocionalmente em processo de aceitação e negação da patologia da criança.

Assim, os resultados identificam que as orientações fisioterapêuticas são realizadas com freqüência em casa e que as respostas esperadas pelos familiares nem sempre são satisfatórias, mas os mesmos conseguem identificar os ganhos das crianças quando auxiliadas em casa.

As mães que receberam maior número de orientações realizam as atividades com mais segurança, reforçando a importância desta atividade no estabelecimento da interação do pro- fissional com a família. Constatamos que a maioria dos familiares não está totalmente esclarecida quanto às limitações da criança, ou apresenta-se em processo de aceitação e/ou negação da patologia da criança. Quaisquer das duas situações constituem indicativos da necessidade de uma atuação mais efetiva por parte dos profissionais junto às mesmas.

A família necessita do apoio profissional, de orientação e também de ser ouvida em suas dúvidas e necessidades. E mesmo defronte as incapacidades, se deve estimular e incentivar a família para a realização das atividades no domicílio, pois o ambiente, o carinho e atenção da família são benéficos para reforçar o vínculo com a criança, o que por sua vez favorece o seu desenvolvimento global.

\section{REFERÊNCIAS}

1. Rotta NT. Paralisia Cerebral, novas perspectivas terapêuticas. J Ped 2002; 78 (supl.1):48-54.

2. Mancini MC, Fiúza PM, Rebelo JM, Magalhães LC, et al. Comparação do desempenho de atividades funcionais em crianças com desenvolvimento motor normal e crianças com paralisia cerebral. Arq Neuropsiquiatr 2002; 60(2-B):446-452.

3. Schwartzman JS. Paralisia Cerebral. Arq. Bras Paralisia Cerebral 2004; 1(1):4-17.

4. Campos D, Santos DCC. Controle postural e motricidade apendicular nos primeiros anos de vida. Fisioterapia em Movimento 2005;18(3):71-77.

5. Shepherd RB. Fisioterapia em Pediatria. $3^{\text {a }}$ ed. São Paulo: Santos, 1996.
6. Finnie NR. A importância da comunicação entre os pais e profissionais. In: Finnie NR (Org.). O manuseio em Casa da Criança com Paralisia Cerebral. $3^{a}$ ed. São Paulo: Manole, 2000, p.03-07.

7. Oliveira SL. Tratado de Metodologia Científica. $2^{\mathrm{a}}$ ed. São Paulo: Pioneira, 1999.

8. Moura EW de, Silva PAC. Fisioterapia: Aspectos clínicos e práticos da reabilitação. São Paulo: Artes Médicas, 2005.

9. Rotta NT. Aspectos da relação médicopaciente em neuropediatria. Neurobiol. 1983; 46(3):301-8. 
10. Moura EW de, Silva PAC. Fisioterapia: Aspectos clínicos e práticos da reabilitação. In: Ozu MHU, Galvão MC dos S. Fisioterapia na paralisia cerebral, São Paulo: Artes Médicas, 2005, p. 27-46.

11. Voyer P, Roien C. Psicologia atual e desenvolvimento da criança. São Paulo: Manole, 1990.

12. Poresky RH, Henderson ML. Infant's mental and motor development: effects of home environment, maternal attitudes, marital adjustment and socioeconomic status. Percept Mot Skills 1982; 53:695-702.

13. Andraca I, Pino P, La Parra A, Marcela RF. Factores de riesgo para el desarrollo psicomotor en lactentes nascidos en optimas condiciones biológicas. Rev Saúde Púb 1998; 32(2):134-47.

14. Levitt S. O Tratado da Paralisia Cerebral e do Retardo Motor. $3^{\text {a }}$ ed. São Paulo: Manole, 2001.

15. Bee H. A criança em desenvolvimento. In: Bee H. (Org). Desenvolvimento atípico. $9^{\mathrm{a}}$ ed. Porto Alegre: Artmed, 2003.

Recebido em: 20/08/2008 Modificado em: 30/10/2008 Aceito em: 22/12/2008 